\title{
Multi-Modal Definite Clause Grammar
}

\author{
Hideo Shimazu, Seigo Arita, and Yosuke Takashima \\ Information Technology Research Laboratories, NEC Corporation \\ 4-1-1 Miyazaki, Miyamac Kawasaki, 216 Japan \\ \{shimazu, arita, yosuke\}@joke.cl.nec.co.jp
}

\begin{abstract}
This paper describes the first reported grammatical framework for a multimodal interface. Although multimodal interfaces offer the promise of a flexible and user friendly means of human-computer interaction, no study has yet appeared on formal grammalical frameworks for them. We have developed MultiModal Definite Clause Grammar (MM-DCG), an extension of Definite Clause Grammas. The major features of MM-DCG include capability to handle an arbitrary number of nodes and temporal information in grammar rules. Further, we have developed MM-DCG translator to transfer rules in MM-DCG in to Prolog predicates.
\end{abstract}

\section{Introduction}

This paper describes the first reported grammatical framework for a multimodal interface. Specifically, the authors have developed MM-DCG (Multi-Modal Definite Clause Grammar), an extension of 1)CG [Pereira and Warren, 1980] for multimodal input processing. The major features of MM-DCG include capability to handle an arbitrary number of modes and temporal information in grammar rules.

The motivation behind this research was two-fold. First, the extension to multimodal was found to be the minimum requirement for natural language interface systems to be installed in real applications. Wo have developed natural language interface for relational database (RDB) [Shimazu ot. al., 1992; Arila et. al., 1992a; Arita et. al., 1992b]. Spolen user queries are transformed into SQL specifications, and dispatchod to RDBMS. The retrieved results are displayed at a com puter terminal. The results include not only table forms but also picture images, like Figure 1 . When users see picture images on the terminal, they naturally want to generate following queries by referring to such picture images. For example, they want to say, "Show me the interior of this one" or "Are there the same type of cars as this car" while pointing at a specific picture on the display. If such multi-modal utterances be acceptable, the natural language interface will be more practical

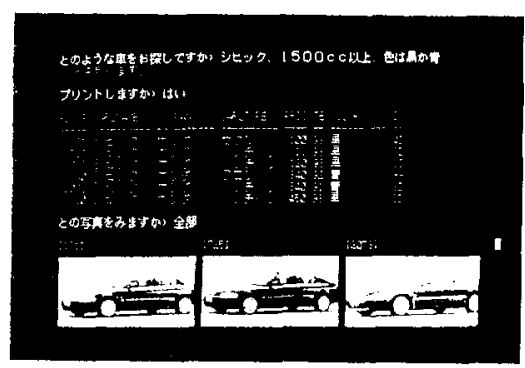

Figure 1: Natural Language Interface Screen Image

enough to be used in many real world applications.

Second, no study has yet appeard on developing formal grammatical framework for multi-modal interfaces. Although there have been many researches on multimodal systems, these systems are built as task-specific expert systems. The capability of such systems to process multi-modal inputs is too limited to interpret complex multi-modal expressions. This is mainly duc to the fact that they have not developed their systems on formal grammatical framework for multi-modal interfaces.

MM-DCG is the first reported grammatical framework for a multimodal interface. Multi-modal input processing rules can be written in MM-1)CG simply and effectively. Rules in MM-DCG are translated into Prolog predicates easily.

\section{Multi-Modal Input Processing}

Consider a query example to a multi-modal interface with a screen image like Figure 1. A user states "Can this, attach this," pointing at a picture on the screen and clicking the mouse during the first "this" and then choosing an item from a menu during the second. The system must realize that the first point is to a specific automobile and the second is to the menu item "CD player". After integrating the two mouse pointing cvents into the two "this" in the utterance, the system must create an intemal representation of this query that conforms to SQI, specifications. In this example, even if the order of the two mouse clicking events is opposite, 
the system must generate the same $S Q L$ specificalion, but the interpretation will be more diflicult. In order to interpret such complex combinations of multi-modal inputs, the following recuirements exist:

(1) Modes should be interprotod oqually and independently. In conventional multi-noodal systens, natural language mode plays a major role, and other modes such as mouse input mode are auxiliary. Iuputs of auxiliary modes are merged in to corresponding nat ural language expressions in a surface level, and the merged natural language query is interpeted by conventional natural language parsers. 'Therefore, variely of accepted multi-modal expressions is very limited.

However, If each node is treated with the sante manner as that, of natural language mode, syntix and somantics of inputs of each mode are defined with grant mar formulation. Thus, complex expressions can be defined declaratively and more casily

(2) Mode interpretation should be referred to one another. Inputs of each mode should be interpreted independently. However, the interpretation of such inputs should be refored by other mode interpretations. There are ambiguties which are solved only by integrating partial interpretations of related nodes. For example, if user states "this calr", poinling at an object which is overlapped on the car object, the ambiguity of the object pointing must be solved by comparing tho two mode interpretations.

(3) Mode interpretation should handle temporal information. Temporal information of inputs, such as input arriving time, interval between lwo inputs, plays an important role to interpret mulli-modal inputs. Consider an example that a user states "How much is this car", and points at, a car picture a little after the utterance. If the interval is three seconds, the pointing event should be integrated with "this car" in the ut. terance. However, if the interval is three minntes, the event should not be integrated.

\section{MM-DCG Design Decisions}

This section describes major design decisions made in developing MM-DC(G. Because MM-1)C(i is a superset of DCG, everything possible in DCG is also possible in MM-DCG. However, two major extensions are provided

\subsection{Receiving Multiple Input Streans}

MM-1)CG can receive arbitiary unmbers of diflecent input streans, while DCG receives only one. Wach mode is assigned an individual stream. Thorefore, a single grammar rule in MM-1)C( can allow the coexistence of grammatical categories in different modes, thus allowing for their integration. In addition, context sensitive information can be interchanged anong categories of different modes in a single puke. figure 2 illustrates a multi-modal input processing nodulo which accepts three independent streams.

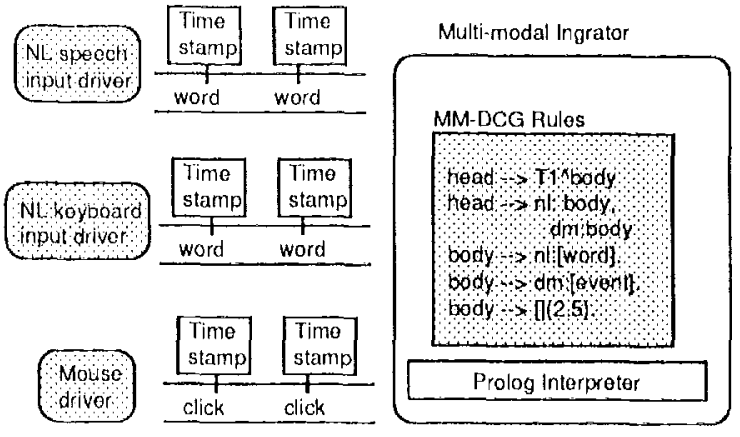

Figure 2: Multi-modal lnput Processing Module

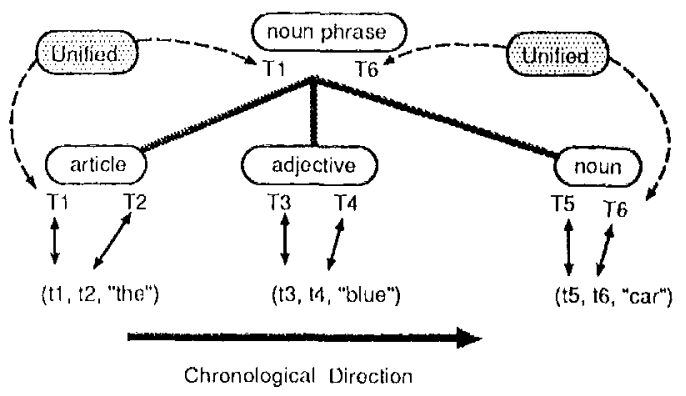

figure 3: 'Tine Calculation of Instantiated Semantic Categories

\subsection{Calculating the Instantiated Time of Grammatical Categories}

Inputs of a single mode invariably have ordering relations among them. $\Lambda$ parser like DCG uses such order relations to analyze syntax, semantics, and pragmatics. Inpuls of different modes, however, have no inherent ordering relations. Therefore, MM-DCG requires the attachment of both the beginning time and the end time to each individual piece of inpul, data. MM-DCG automatically calculates the beginning time and the end time of any level of grammatical categories generated during parsing.

MM-DCG translatol antomatically generates the code which calculates the beginning and end times of any body goal in a gramnar rule. The translator generates two extra arguments to store the beginning time and ond timo into each head and body goals in MMDCG rules. The begimning time argument of the head is unified with the beginning time argument of the first body goal. 'The end time argument of the head is unified with the end time argument of the last body goal. Figure 3 shows the argument organization of noun_phrase rule.

Thus, for example, if a noun phrase category is instantialed by parsing "hlo blue car", the beginning time of the instantiated category becomes equal to the beginning time of "the", and the end time of the category is equal to the end time of "car". 


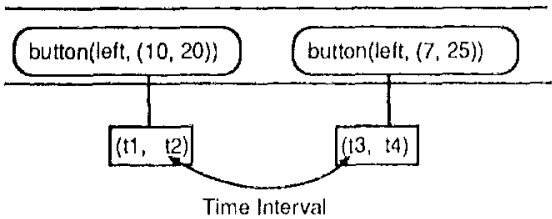

Figure 4: 'Timeout Concept

MM-DCG requires any input from every mode to have beginning and end times. Thus, cach item in an input sequence will have the following structure:

input (beginning-time, end-time, <actual input>)

which means that the actual input wiss in putted from start-time and completed at end-time. Adling of this time information is easy for any of the sorts of inpul modes we are considering (i.e. speech recognition, keyboard inputs, mouse pointing, etc).

One other important item of notation: If a variable is explicitly bound within a goal, the variable returns the beginning and end times of the goal in the form of a functor. Thus,

\section{Time^goal}

means that "if goal sueceeds, the beginuing time and end time of the goal are returned in the variable Time." Using the time information of instantiated categories, rule writers can define chronological constraints among categories, for example, the following description expresses a constraint that pronoun calcegory and pointing category must be both instantiated within a five seconds,

T1"pronoun, T2"pointing,

\{Diff is T2 - T1, Diff $<5\}$

\subsection{Defining Timeont in Rules}

Timeout is a constraint of intervals between an input. and its succeeding input of a stream (Seo ligure 1). If an interval between inputs of a strean becones larger than a threshold defined in grammat rules, the timoout occurs, and the stream is regarded omply temporarily although there still exist inputs in it.

The following points rule means that "Receive mouse clicking inputs while the interval between two inputs is less than 5 seconds or until a stream becomes null, then return the list of the inputs"

points ([]) $\rightarrow$ mouse: [] $(5.0)$. points([Pt | Pts]) $\rightarrow$ point(Pt), points(Pts) point(LOC) $\rightarrow$ mouse: [button(left, LOC)].

\section{Rules Written in MM-DCG}

\subsection{Syntax}

MM-DCG syntax extends DCG in the following ways:
- A body goal may or may not be specified its consuming stream:

If a body goal consumes inputs from specific streams, the goal must be accompanied by the stream names. For example, the following rule

noun_phrase $\rightarrow$ keyboard:pronoun.

means that "if the pronoun category is found which is generated by inputs from the keyboard stream, noun_phrase is found." If a body goal is not accompanied by any stream name, the goal is regarded as consuming some amount of inputs from all modes. For example, the following rule

noun_phrase $\rightarrow$ noun.

means that "if the noun category is found which is generated by inputs from certain streams, noun_phrase is found."

- A terminal symbol should always be accompanied by a specific stream name:

for example, the following rule

pointing $\rightarrow$ mouse: [button(left, $\operatorname{loc}(X, Y)]$.

moans that "if a functor button(left, $\operatorname{loc}(X, Y)$ ) is

found at the mouse stream, pointing is found".

\subsection{Rule Examplo}

To demonstrate how MM-DCG rules are written, this section describes a simple grammar needed to handle "object" with multi-modal inputs.

Figure 5 shows the definition of "object". A rule writer defines existing streams specifically using a unit clause, active stream/1. "Object" arc specified by using either one of the above modes or their combinations.

The first object/1 definition interprets natural language specifications such as "the blue car". "The second object/1 interprets a mouse clicking which points at a specific graphical object on the display. 'The third object/1 definition interprets a combination of a natural language utterance and a mouse pointing, such as stating "the blue car" while pointing at a graphical object on the display. A natural language utterance is interpreted at the noun_phrase body goal, and the identified object is bound to Objl. A mouse pointing event is interpreted at the pointing body goal, and the identified object is bound to Obj2.

Then, Obj1 and Obj2 are compared their values in a Prolog predicate enclosed inside curly brackets $\{$ and \}. Both variables should be equal. If not, because the interpretation of noun _phrase or pointing must be wrong, backtracking occurs.

As seen above, a single grammar rule in MM-DCG can allow the coexistence of grammatical categories in different modes, thus allowing for their integration. In addition, temporal and context sensitive information can be interchanged among categories of different modes in a single rule. 


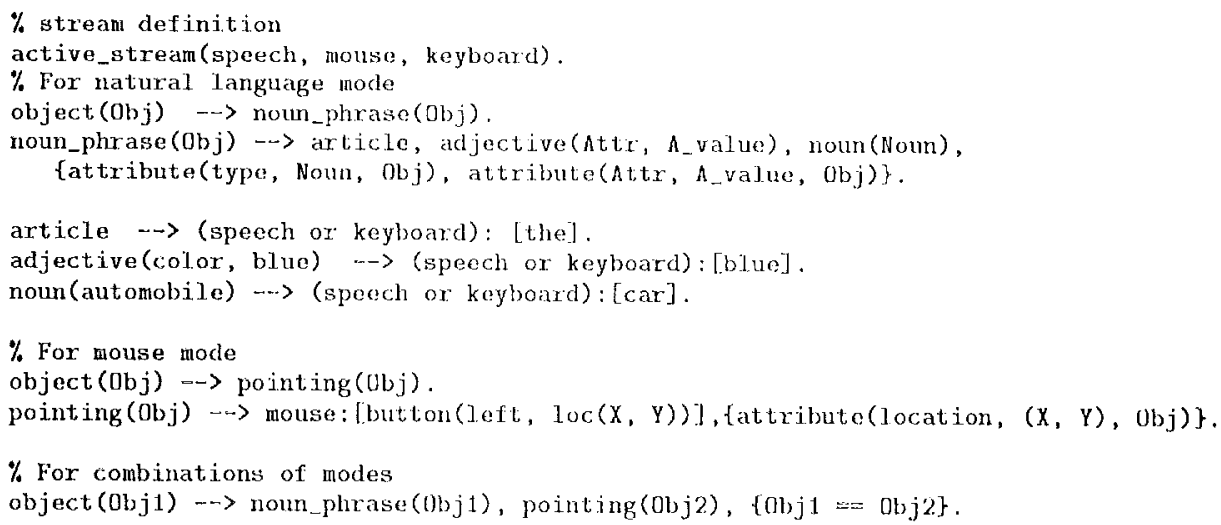

Figure 5: Cirammar Description bixample Using MM-DCG

\section{Translating MM-1)CG into Prolog}

This section describes tramblation tecturicumes of $\mathrm{MM}$ DC(r rules into Prolog predicalles. first. we explain the translation method of MM M)( ( i rules with a single stream. Even in the single strean case, MM-l)c (;

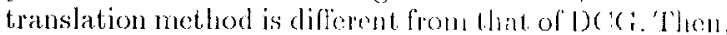
the translation technigue with multiple streans is explained.

5.1. MM-DCG 'Translation for a Single Stream A head and body gonls in a granumar mole are translated into a predicate witl four extrat argunents two for the beginning time and the end time and lwo for expressing a consumed input stream. 'l'he latter two arguments ate the same as the generated argumentis when l)(: (x) rules are translated into l'rolog predicales.

The beginning time argument of the head is unified with the beginning time argument of the first, body goal, and the end time argument of the head is unified with the end time of the last body goal. f'ol example, the following MM-I)(Ci rule (for a single streame)

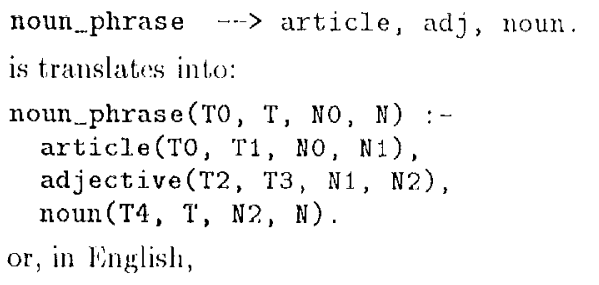

There is a noum-phrase between $N O$ and $N$ if there is an article between NO and Nl, and if there is an adjective between $\mathrm{N} /$ and $\mathrm{N} 2$, and if there is a noun betwern $N 2$ and $N$. 'The nounphrase starts at 'TO, and ends at T'. The anticle starts at ' $T 0$, and conds at 'Th. The adjoctive starts at 'T2, and ends al 'T3. The nomm stath at Th, and ends at' 1

$\Lambda$ rule with a terminal symbol is translated into at unit clause. For example, noun $\rightarrow \rightarrow$ keyboard: [window].

translates into:

noun (T's , Te, [input(Ts, Te, "window")।N] , N).

$A$ functor input $/ 3$ is inserted into the third argument forming the input stream of the predicate. The third arguncut of the functor input/3 is the actual iuput item, the "window" string in this example.

I'le first and second argument of input/3 is unified with the first and second argument of this mut clanse lespectively. "Therefore, if a string "window" is input via the keyboard stream, the noun category is instantiated, and the beginning and end time of the noun category is the same as the start and end time attached to the "window" input.

\subsection{Extension to Arhitrary Number of Streams}

lixtension fron a single stream to multiple streams is easy. Wach strean needs four extra arguments - two for timing information and two for expressing a consumed input strcam. 'Thus, if there are $n$ modes, $4 x$ arguments are aclded into head and goals arguments.

lior cxample, if there are two streams, the noun_phrase defintion in the previous section is translated into the following prolog predicates with eight $(2 \times 4)$ extra argunent.s:

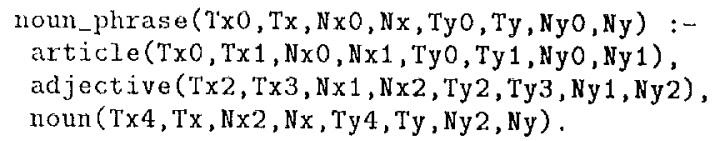

\subsection{Fxtractions of Temporal Iuformation}

If there is a variable bindiug within a goal like,

Time"goal

the goil is transtated into a conjunction of two body goals (for a single mode):

(goal (TO, T1, No, N), Time $\left.=\left(T^{\prime} O, T 1\right)\right)$ 
If there exist $n$ streams, the variable Time is bound to a list of $n$ time pairs, such as for two modes:

(goal ( TxO, Tx1, NxO, Nx1, TyO, Ty1, NyO, Ny1), Time $=[(\mathrm{Tx} 0, \mathrm{Tx} 1),(\mathrm{Ty} 0, \mathrm{Ty} 1)])$

\section{Related work}

The idea of understanding multi-modal iuputs in conjunction with each other, as presentcd in this paper, is not particularly new. The idea of a multi-modal input combining motions and pointing has been cxplored in a number of contexts. The classic 1980 paper "Put-ThatThere" [Bolt, 1980] describes an early system that procedurally combined voice and gesture inputs. This idea was further explored in terms of integrating natural language and pointing by [Hayes, 1988], who related multimodal inputs to anaphoric reference in natural language processing, particularly to the work of [Grose, 1977] and [Sidner, 1979]. Recent work in the design of direct manipulation interfaces has also explored the notion of integrating a set of diverse inputs. Other papers exploring multimodal interfaces include [ $\Lambda$ llgayer ot. al, 1989; Cohen et. al. 1989; Cohen, 1991; Kobsa et. al., 1986; Wahlster, 1989]. Most of this work, however, has focused on the application of the ideas, and not on the principles for integrating the different imputs. ${ }^{1}$

\section{Conclusion}

In this paper, we have proposed the use of a grammar for dealing with inpul events in a multi-modal user interface. We proposed MM-DCC, a novel grammatical framework for a multimodal interface. $M M-1)(G$ is all extension of DCG for mulli-modal iupuls processing. The major features of MM-DCG include capability to handle an arbitrary number of modes and temporal information in grammar rules. We showed its use for a simple example. The translation technique of the MMDCG rules in to Prolog predicates was also presented An initial implementation of MM-DCG has been developed at NEC Corporation, and is currently being used for the development of a prototype multimodal interface.

\section{References}

[Allgayer et. al., 1989] Allgayer, J., Jansen-Winkeln, R., reddig, C., and Reithing N.,

[Arita et. al., 1992a]

Arita, S., Shimazu, H., and Takashima, Y', "Portable Natural Language Interface", Proc. of the 8t, Human Interface Symposium, 1992, (in Japancse).

[Arita ct. al., 1992b]

Arita, S., Shimazu, H., and Takaslima, Y., "Simple + Robust $=$ Pragmatic: A Natural Tanguage Query Processing Model for Card-type Databases", Proc. of the 13th Annual Conference of the Cogntitive Scicnce Society, 1992.

\footnotetext{
${ }^{1}$ A survey of this work is beyond the scope of this paper, the interested reader is directed to the review in [Shneiderman, 1991].
}

[Bolt, 1980] Bolt, R.A., "Put-That There: Voice and Gesture at the Graphics Interface", Computer Graphics 14, 3,1980 .

[Clocksin and Mellish, 1981] Clocksin, W.F. and Mellish, C.S., "Programming in Prolog", Springer-Verlag, 1981.

[Cohen et. al. , 1989] Cohen, P.R., Dalrymple, M., Moran, D.B., Pereira, F.C.N., et al., "Symergistic Use of Direct Manipulation and Natural Language", Proc. of CHI-88, 1989.

[Cohen, 1991] Colien, P.R., "The Role of Natural Language in a Multimodal Interface", 1991 International Sympo sium on Next Generation Human Interface, 1991.

[Grosz, 1977] Grosz, 13. "The representation and use of focus in a system for uuderstanding dialogs," Proc. IJCAI 1977, Boston, MA

[Mayes, 1987] Hayes, P.J., "Steps towards Integrating natural Language and Graphical Interaction for Knowledgebased Systems", Advances in Artificial Intelligence- II, Elsevier Science Publishers, 1987.

[Hayes, 1988] IIayes, P.J., "Using A Knowledge Base To Drive $A n$ Expert System Interface With $\Lambda$ Natural Ianguage Component," in J. IIendler (ed.) Expert Systems: The User Interface, Ablex Publishing, 1988.

[Kobsa et. al., 1986] Kobsa, A., Al]gayer, J,, Reddig, C. Reithing, Nl, Sclimmauks, D., Harbusch, K., and Wablster, W, "Combining Deictic Gestures and Natural Language for Referent Iclentification", Proc. of COLING-86, 1986 .

[Pereira and Warren, 1980] Pereira, F., and Warren, D.IT.D., "Defunite Clause Grammars for Language Analysis - A survey of the Formalism and a Comparison with Augmented Transition Networks", Artificial Intelligence, vol. 13, no. 3, 1980.

[Shimazu et. al., 1992] Shimazu, Il, Arita, S., and 'Takushima, Y., "Design Tool Combining Keyword Analyzer and Case-Based Parser for Developing Natural Language DataBase Interfaces", Proc. of COL.ING-92, 1992.

[Shuciderman, 1991] Designing The User Interface, Addison Wesley Publ, Reading, MA.

[Sidner, 1979] Sidner, C. Towards a computational theory of definite anaphora comprohension in English Discourse, TR-537, MIT AI liab, Cambridge, Ma.

[Wahlster, 1989] Wahlster, W., "User and discourse models for multimodal communication", in J.W. Sullivan and S.W. Tyler, editors, Intelligent User Interfaces, chapter3, ACM Press Frontiers Scries, Addison Wesley Publishing, 1989 . 